\title{
TRATAMENTO TERCIÁRIO DE EFLUENTE FRIGORÍFICO POR PROCESSO FENTON
}

\author{
F. S. COLOMBARI ${ }^{1}$; A. K. GENENA ${ }^{1}$; D. C. LENHARD ${ }^{2}$; A. CAVALLI ${ }^{2}$ \\ ${ }^{1}$ Universidade Tecnológica Federal do Paraná, Câmpus Medianeira, Programa de Pós- \\ Graduação em Tecnologia de Alimentos \\ ${ }^{2}$ Universidade Tecnológica Federal do Paraná, Câmpus Medianeira \\ E-mail para contato: fernanda_colombari@yahoo.com.br
}

\begin{abstract}
RESUMO - O tratamento adequado de efluentes industriais corrobora com a sustentabilidade do meio ambiente ligada à qualidade do efluente a ser lançado em corpos receptores de água, e pode possibilitar, ainda, que esse efluente seja utilizado como agua de reuso dentro da própria indústria, o que vai de encontro com a preservação da água por meio da redução da captação de recursos hídricos. Dessa forma, o presente trabalho teve como objetivo principal investigar a eficiência da aplicação do processo de oxidação avançada Fenton, capaz de degradar compostos recalcitrantes, como tratamento terciário do efluente de um frigorífico de suínos. Após o tratamento foi possível obter $67,71 \%$ de remoção da DQO, na qual a degradação da matéria orgânica do efluente seguiu a lei cinética de pseudo-primeira ordem, com redução na toxicidade após o tratamento.
\end{abstract}

\section{INTRODUÇÃO}

Os abatedouros e frigoríficos geram uma grande quantidade de efluentes líquidos, que são quase inteiramente orgânicos, dentre os quais estão os poluentes orgânicos persistentes, como fármacos de uso humano e veterinário em geral, pesticidas, produtos de higiene pessoal, metabólitos de detergentes, entre outros (Gibs et al., 2007). Estes efluentes são normalmente tratados por processos biológicos convencionais, os quais são eficientes na remoção da matéria orgânica, porém, não são capazes de degradar os poluentes orgânicos persistentes presentes. Segundo Bila e Dezotti (2003), muitos fármacos residuais são resistentes aos processos convencionais de tratamento da água.

Os POAs (Processos de Oxidação Avançada) têm sido utilizados como alternativa para o tratamento desses compostos orgânicos persistentes. Estes processos caracterizam-se pela produção do radical hidroxil $(\bullet \mathrm{OH})$, capaz de mineralizar contaminantes a dióxido de carbono e água, ou oxidá-los a compostos biodegradáveis e não tóxicos (Andreozzi et al., 1999). Dentre os POAs está o processo Fenton que se baseia na geração de $\cdot O H$ a partir da decomposição de peróxido de hidrogênio catalisada por íons ferrosos $\left(\mathrm{Fe}^{+2}\right)$ em condição ácida (Britto; Rangel, 2008).

Vários autores têm estudado a eficiência do tratamento de efluentes de indústrias alimentícias por meio do processo Fenton, seja como tratamento principal (Lucas; Peres 2009; Martins; Rossi; Quinta-Ferreira, 2010), seja como tratamento terciário (Mandal et al., 2010; Zorpas, Costa, 2010; Sánchez Peréz et al., 2014). 
O presente trabalho tem como objetivo, investigar a eficiência do uso do processo Fenton no tratamento terciário de um efluente agroindustrial.

\section{MATERIAL E MÉTODOS}

\subsection{Materiais}

O efluente de estudo, proveniente de uma indústria frigorífica situada na região Oeste do Paraná, foi coletado após o tratamento biológico realizado pelo frigorífico. Os reagentes utilizados para realização dos experimentos foram, sulfato ferroso heptahidratado P.A $\left(\mathrm{FeSO}_{4}, 7 \mathrm{H}_{2} \mathrm{O}\right)$, peróxido de hidrogênio 35\% P.A $\left(\mathrm{H}_{2} \mathrm{O}_{2}\right)$, ambos da Êxodo Científica. Todos os demais reagentes utilizados foram de grau analítico.

\subsection{Reator Químico}

Os ensaios para o tratamento do efluente foram conduzidos no modo batelada sob agitação constante (100 rpm), em equipamento Jar Test (Poli Control, modelo Floc Controll III) de seis provas (béckers de polietileno com capacidade de $400 \mathrm{~mL}$ cada).

\subsection{Processo Fenton}

Ao efluente $(400 \mathrm{~mL})$ adicionou-se íons $\mathrm{Fe}^{+2}$ na concentração desejada, e procedeu-se ao ajuste do $\mathrm{pH}$ da mistura reacional $\left(\mathrm{NaOH}\right.$ ou $\left.\mathrm{H}_{2} \mathrm{SO}_{4}\right)$, seguido da adição de $\mathrm{H}_{2} \mathrm{O}_{2}$ na concentração desejada. Na primeira etapa do processo Fenton (oxidação química), a mistura reacional foi agitada por $1 \mathrm{~h}$ e, $\operatorname{logo}$ em seguida, realizou-se a segunda etapa do processo (coagulação química), na qual o $\mathrm{pH}$ da solução foi ajustado sob agitação para a faixa desejada, e então, a agitação foi interrompida. A solução permaneceu em repouso por $1 \mathrm{~h}$ para sedimentação dos flocos formados (lodo). Ao final do processo, após a separação do lodo, obteve-se o efluente tratado (sobrenadante).

\subsection{Planejamento Experimental}

Com base em testes preliminares, a influência dos parâmetros operacionais para o tratamento do efluente com o processo Fenton, foi investigada baseada na eficiência de remoção da DQO, utilizando como ferramenta o Delineamento Central Composto Rotacional (DCCR), com 04 (quatro) pontos axiais e 03 (três) pontos centrais, totalizando 11 ensaios. No qual as variáveis independentes utilizadas, com suas respectivas faixas foram: pH de coagulação $(5,5$ á 8,0$)$ e razão $\left[\mathrm{Fe}^{+2}\right] /\left[\mathrm{H}_{2} \mathrm{O}_{2}\right](0,30$ á 0,80$)$. Sendo fixado o pH de reação em 3,0 e razão $\left[\mathrm{H}_{2} \mathrm{O}_{2}\right] /[\mathrm{DQO}]$ de 1,5 .

\subsection{Determinações analíticas}

Para determinar os parâmetros físico-químicos do efluente em estudo foram utilizadas as metodologias segundo os procedimentos descritos pelo Standard Methods for the Examination of Water and Wastewater (APHA, 1999). A cor real foi mensurada pelo método platina-cobalto, utilizando o espectrofotômetro HACH (DR 2800) no comprimento de onda de $455 \mathrm{~nm}$. A DQO foi determinada pelo método colorimétrico de refluxo fechado no comprimento de onda $600 \mathrm{~nm}$. A turbidez foi determinada pelo método nefelométrico, utilizando um turbidímetro (Poli Control, Ap 200), sendo que, os resultados foram expressos em NTU (Unidades Nefelométrica de Turbidez), e o $\mathrm{pH}$ foi medido pelo método potenciométrico utilizando um $\mathrm{pHmetro}$ digital (Hanna $\mathrm{pH} / \mathrm{mV}$ meter). 


\subsection{Análise de $\mathrm{H}_{2} \mathrm{O}_{2}$ Residual}

$\mathrm{O} \mathrm{H}_{2} \mathrm{O}_{2}$ residual nas amostras de efluente tratadas foi determinado por meio do método iodométrico catalisado com molibdato de amônio (Kolthoff, 1920). As correções da interferência do $\mathrm{H}_{2} \mathrm{O}_{2}$ na DQO foram realizadas de acordo com Kang et al. (1999).

\subsection{Determinação de Ferro Total}

A determinação da concentração de ferro total foi realizada pelo método da espectrometria de absorção atômica por chama, segundo os procedimentos descritos pela NBR 13815/1997 (ABNT, 1997).

\subsection{Toxicidade}

A evolução da toxicidade no tratamento do efluente pelo processo Fenton nas melhores condições operacionais determinadas no planejamento operacional foi realizada por meio do ensaio com o microcustáceo Artemia salina, baseado na técnica descrita por Meyer et al. (1982). A diluição utilizada no bioensaio foi de 0,7:2,0 de amostra:solução salina.

Procedeu-se em paralelo com um ensaio Fenton, no qual ao invés do efluente, utilizouse água destilada, para eliminar a interferência dos reagentes na mortalidade do microcrustáceo.

\subsection{Cinética de Degradação}

Para o estudo da cinética de degradação de matéria orgânica (DQO), procedeu-se com ensaio Fenton nas melhores condições operacionais determinadas no planejamento experimental, no qual amostras foram retiradas periodicamente durante o tempo reacional total de 20 min, e submetidas à análise de DQO e $\mathrm{H}_{2} \mathrm{O}_{2}$ residual. A análise dos dados cinéticos foi realizada por meio do método integral, a partir da suposição de lei cinética de pseudo-primeira ordem. Os ensaios foram realizados em duplicata.

\section{RESULTADOS E DISCUSSÃO}

\subsection{Caracterização do efluente}

Os resultados das análises físico-químicas realizadas para caracterizar o efluente de estudo estão apresentados na Tabela 1.

Tabela 1 - Resultados da caracterização do efluente

\begin{tabular}{ccc}
\hline Parâmetros & Valor & Unidade \\
\hline Cor real & $719,67 \pm 1,53$ & $\mathrm{mg} \mathrm{PtCo} \mathrm{L}^{-1}$ \\
DQO & $129,17 \pm 2,05$ & $\mathrm{mg} \mathrm{O}_{2} \mathrm{~L}^{-1}$ \\
Turbidez & $50,10 \pm 0,50$ & $\mathrm{NTU}$ \\
$\mathrm{pH}$ & $7,63 \pm 0,01$ & - \\
\hline
\end{tabular}




\subsection{Planejamento experimental}

Com o planejamento DCCR foi possível obter máxima remoção de DQO de 67,71\%, nas condições de pH de coagulação 7,63 e razão $\left[\mathrm{Fe}^{+2}\right] /\left[\mathrm{H}_{2} \mathrm{O}_{2}\right]$ de 0,800 . Valor semelhante de $66,98 \%$ de remoção de DQO foi observado no tratamento com pH de 5,50 e razão $\left[\mathrm{Fe}^{+2}\right] /\left[\mathrm{H}_{2} \mathrm{O}_{2}\right]$ de 0,55 , não tendo diferença significativa $(\mathrm{p}<0,05)$ entre as duas condições, como pode ser verificado na Tabela 2.

Tabela 2- Planejamento DCCR para o estudo da eficiência de remoção da DQO, no tratamento Fenton do efluente frigorifico com resultados (níveis codificados e reais)

\begin{tabular}{cccc}
\hline Variáveis/níveis & pH de Coagulação & {$\left[\mathbf{F e}^{+2}\right] /\left[\mathbf{H}_{2} \mathbf{O}_{2}\right]$} & \% Remoção \\
\hline $\mathrm{T} 1$ & $-1(5,86)$ & $-1(0,373)$ & 42,02 \\
$\mathrm{~T} 2$ & $+1(7,63)$ & $-1(0,373)$ & 34,93 \\
$\mathrm{~T} 3$ & $-1(5,86)$ & $+1(0,727)$ & 41,25 \\
$\mathrm{~T} 4$ & $+1(7,63)$ & $+1(0,727)$ & 55,98 \\
$\mathrm{~T} 5$ & $-1,41(5,50)$ & $0(0,550)$ & 66,98 \\
$\mathrm{~T} 6$ & $+1,41(8,00)$ & $0(0,550)$ & 36,90 \\
$\mathrm{~T} 7$ & $0(6,75)$ & $-1,41(0,300)$ & 54,32 \\
$\mathrm{~T} 8$ & $0(6,75)$ & $+1,41(0,800)$ & 67,71 \\
$\mathrm{~T} 9$ & $0(6,75)$ & $0(0,550)$ & 58,59 \\
$\mathrm{~T} 10$ & $0(6,75)$ & $0(0,550)$ & 58,36 \\
$\mathrm{~T} 11$ & $0(6,75)$ & $0(0,550)$ & 58,62 \\
\hline
\end{tabular}

Outros estudos obtiveram resultados semelhantes. Mota e Brito (1999) obtiveram 50\% de remoção no processo Fenton após o tratamento biológico em efluente de indústria química de cola e verniz. Elmolla e Chaudhuri (2009) observaram remoção de 54,3\% em efluente com resíduos de antibióticos. Neste estudo o reagente Fenton foi aplicado como um pré-tratamento, auxiliando o tratamento biológico.

Para melhor entender as condições de maior eficiência na remoção, foram calculados os coeficientes de regressão, que estão apresentados na Tabela 3. Apesar de todas as variáveis não terem apresentado significância ao nível de 5\%, é interessante ressaltar que a variável $\mathrm{pH}$ de coagulação apresentou efeito negativo, sendo possível utilizar o nível inferior. Um resultado semelhante foi observado por Kang et al. (1999) em estudo realizado com chorume tratado biologicamente. A maior eficiência ocorreu com pH de coagulação na faixa entre 3 a 6.

Em relação a variável $\left[\mathrm{Fe}^{+2}\right] /\left[\mathrm{H}_{2} \mathrm{O}_{2}\right]$ o efeito foi positivo, isso ocorreu devido o $\mathrm{Fe}^{+2}$ ser o reagente limitante da reação de Fenton, quando maior a concentração e íons ferrosos, maior a \% de remoção de DQO. Porém é necessário estabelecer um limite na concentração de ferro para que não ocorra um excesso desses íons no efluente e venha comprometer a qualidade do efluente tratado. 
Tabela 3 - Coeficientes de regressão para \% de Remoção de DQO obtidos no DCCR

\begin{tabular}{cccccc}
\hline Fatores & Efeitos & $\begin{array}{c}\text { Coeficiente } \\
\text { de Regressão }\end{array}$ & $\begin{array}{c}\text { Erro } \\
\text { Padrão }\end{array}$ & Valor $\boldsymbol{t}$ & P - valor \\
\hline Média & 58,5730 & 58,57298 & 6,570524 & 8,91451 & 0,000296 \\
$\mathbf{X}_{\mathbf{1}}(\mathbf{L})$ & $-8,7158$ & $-4,35792$ & 4,029654 & $-1,08146$ & 0,328870 \\
$\mathbf{X}_{\mathbf{1}}(\mathbf{Q})$ & $-13,1737$ & $-6,58684$ & 4,808426 & $-1,36985$ & 0,229045 \\
$\mathbf{X}_{\mathbf{2}}(\mathbf{L})$ & 9,8169 & 4,90846 & 4,029654 & 1,21808 & 0,277530 \\
$\mathbf{X}_{\mathbf{2}}(\mathbf{Q})$ & $-4,0399$ & $-2,01996$ & 4,808426 & 0,42009 & 0,691863 \\
$\mathbf{X}_{\mathbf{1}} \mathbf{X}_{\mathbf{2}}$ & 10,9095 & 5,45475 & 5,690308 & 0,95860 & 0,381783 \\
\hline $\mathbf{X}_{1} \mathbf{p}$
\end{tabular}

$\mathrm{X}_{1} \mathrm{pH}$ de Coagulação; $\mathrm{X}_{2}\left[\mathrm{Fe}^{+2}\right] /\left[\mathrm{H}_{2} \mathrm{O}_{2}\right] ; \mathrm{p} \leq 0,05 ; \mathrm{L}$ - termo linear; $\mathrm{Q}$ - termo quadrático.

Neste sentido a partir da determinação do ferro total nas amostras dos tratamentos realizados, verificou-se que a quantidade de ferro presente encontra-se dentro da faixa determinada na legislação para o lançamento de efluentes, que é de $15 \mathrm{mg} . \mathrm{L}^{-1}$ (resolução do CONAMA no 430 de 13 de maio de 2011), viabilizando a utilização da razão $\left[\mathrm{Fe}^{+2}\right] /\left[\mathrm{H}_{2} \mathrm{O}_{2}\right]$ igual 0,8 , sem causar danos ao meio ambiente.

Conforme apresentado na Tabela 4 da análise de variância (ANOVA), o modelo gerado não pode ser considerado válido dentro do intervalo de confiança de $95 \%$, pois o fator resultante da análise da ANOVA Fcalculado (1,363) foi menor que o Ftabelado $(4,387)$, concluindo que o modelo matemático não se ajustou aos dados experimentais. Esse fato é plausível uma vez que se trata de um efluente industrial real, com uma matriz de compostos complexa.

Tabela 4 - ANOVA para porcentagem de remoção de DQO obtidos no DCCR

\begin{tabular}{ccccccc}
\hline $\begin{array}{c}\text { Fonte } \\
\text { Variação }\end{array}$ & $\begin{array}{c}\text { Soma dos } \\
\text { Quadrados } \\
\text { (SQ) }\end{array}$ & $\begin{array}{c}\text { Graus de } \\
\text { Liberdade } \\
\text { (GL) }\end{array}$ & $\begin{array}{c}\text { Quadrado } \\
\text { Médio } \\
\text { (MQ) }\end{array}$ & Fcalc & Ftabelado & p-valor \\
\hline Regressão & 735,795 & 5 & 147,159 & 1,363 & 4,387 & 0,354553 \\
Resíduos & 646,780 & 6 & 107,963 & & & \\
Total & 1383,575 & 11 & & & & \\
\hline $\mathrm{R}^{2}=52,15 \% ; \operatorname{Radj}=43 \%$ & & & & & &
\end{tabular}

Considerando que o objetivo do planejamento foi aumentar a eficiência da porcentagem de remoção da DQO, mesmo que o modelo matemático não tenha se ajustado aos dados experimentais, foi possível chegar a parametros próximos à faixa de otimização (pH de reação 3,0; $\mathrm{pH}$ de coagulação 5,5; razão $\left[\mathrm{H}_{2} \mathrm{O}_{2}\right] /[\mathrm{DQO}] d e$ e 1,5 e razão $\left[\mathrm{Fe}^{+2}\right] /\left[\mathrm{H}_{2} \mathrm{O}_{2}\right]$ de 0,8$)$.

\subsection{Cinética de degradação}

A partir dos dados obtidos experimentalmente em cada ensaio, plotou-se

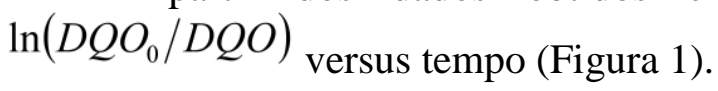




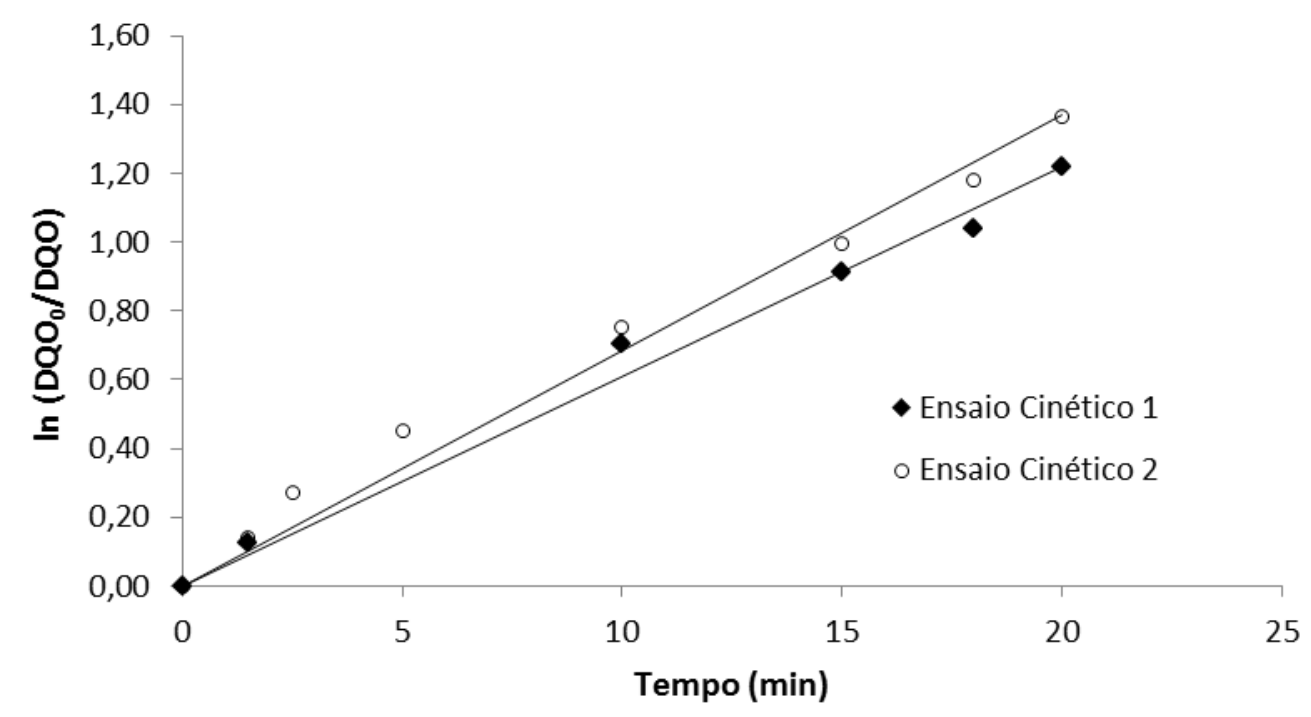

Figura 1 - Cinética de pseudo-primeira ordem para degradação de DQO pelo processo Fenton.

A regressão linear para ambos os ensaios resultou em valores de $\mathrm{R}^{\mathbf{2}}$ (quadrado do coeficiente de correlação amostral) superiores a 0,98 , de forma que se confirmou a suposição inicial de que a degradação da matéria orgânica do efluente pelo processo Fenton seguiu a lei cinética de pseudo-primeira ordem, e a taxa de reação determinada foi de $6,63 \pm$ $0,84 \mathrm{mgO}_{2}$.(L.min $)^{-1}$.

\subsection{Toxicidade}

A partir da avaliação da evolução da toxicidade no decorrer do tratamento do efluente pelo processo Fenton, verificou-se a diminuição da mortalidade com o tempo de reação. Após 60 minutos de reação, a mortalidade foi reduzida em $68 \%$. Esse resultado demonstra que não ocorre a geração de compostos intermediários tóxicos, o que seria um inconveniente no emprego do tratamento proposto.

\section{CONCLUSÃO}

A partir dos resultados obtidos para o planejamento DCCR, verificou-se a eficiência na degradação da matéria orgânica do efluente em estudo por meio do processo Fenton, operando nas condições : pH de reação 3,0; $\mathrm{pH}$ de coagulação 5,50; razão $\left[\mathrm{H}_{2} \mathrm{O}_{2}\right] /[\mathrm{DQO}]$ de

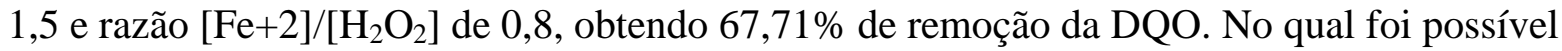
verificar que a cinética de degradação da matéria orgânica do efluente pelo processo Fenton seguiu a lei cinética de pseudo-primeira ordem. Os testes de toxicidade realizados para o efluente tratado indicaram a redução na toxidade do efluente após o tratamento. A partir destes resultados, o tratamento Fenton para efluente agroindustrial pode ser considerado satisfatório, demostrando a aplicabilidade deste processo. 


\section{REFERENCIAS}

ASSOCIAÇÃO BRASILEIRA DE NORMAS TÉCNICAS. NBR 13815: Água Determinação de ferro - Método da espectrometria de absorção atômica por chama. Rio de Janeiro: ABNT, 1997.

APHA. Standard Methods for the Examination of Water and Wastewater, 20th ${ }^{\mathrm{Ed}}$. American Public Health Association, Washington DC, USA. 1999.

ANDREOZZI, R.; MAROTTA, R. Ozonation of p-chlorophenol in aqueous solution. $J$. Hazard. Mat., v. 69(3), p. 303-317, 1999.

BILA, D. M.; DEZOTTI, M. Fármacos no meio ambiente. Quim. Nova, v. 26, n. 4, p. 523530, 2003.

BRASIL. Resolução $n^{\circ} 430$, de 13 de maio de 2011. Dispõe sobre as condições e padrões de lançamento de efluentes, complementa e altera a Resolução no 357, de 17 de março de 2005, do Conselho Nacional do Meio Ambiente, CONAMA.

BRITTO, J. M.; RANGEL, M. D. C. Processos avançados de oxidação de compostos fenólicos em efluentes industriais. Quim. Nova, v. 31, n. 1, p. 114-122, 2008.

ELMOLLA, E.; CHAUDHURI, M. Optimization of Fenton process for treatment of amoxicillin, ampicillin and cloxacillin antibiotics in aqueous solution. J. Hazard. Mat., v. 170(2-3), p. 666-672, 2009.

GIBS, J.; STACKELBERG, P. E; FURLONG, E. T.; MEYER, M.; ZAUGG, S. D.; LIPPINCOTT, R. L. Persistence of pharmaceuticals and other organic compounds in chlorinated drinking water as a function of time. Sci. Total Environ., v. 373, n. 1, p. 240-249, 2007.

KANG, Y. W.; CHO, M. J.; HWANG, K. Y. Correction of hydrogen peroxide interference on standard chemical oxygen demand test. Water Res., v. 33, n. 5, p. 1247-1251, 1999.

KOLTHOFF, I. M. Practische torpassing vas jo-dometrische titraties. Chem. Weekblad, v. 17, p. 197,1920

LUCAS, M. S.; PERES, J. A. Removal of COD from olive mill wastewater by Fenton's reagent: Kinetic study. J. Hazard. Mat., v. 168(2-3), p. 1253-1259, 2009.

MANDAL, T.; DASGUPTA, D.; MANDAL, S.; DATTA, S. Treatment of leather industry wastewater by aerobic biological and Fenton oxidation process. J. Hazard. Mat., v. 180(13), p. 204-211, 2010.

MARTINS, R. C.; ROSSI A. F.; QUINTA-FERREIRA, R. M. Fenton's oxidation process for phenolic wastewater remediation and biodegradability enhancement. J. Hazard. Mat., v. 180( 1-3), p. 716-721, 2010.

MEYER, B. N.; FERRIGNI, N. R.; PUTNAM, J. E.; JACOBSEN, L. B.; NICHOLS, D. E.; MCLAUGHLIN, J. L. Brine Shrimp: A Convenient general bioassay for active plant constituents. Planta Med., v. 45, p. 31-34, 1982.

MOTA, M; BRITO, A. G. Estudo da tratabilidade de um efluente da indústria química por um reactor SBR complementado por reagente de Fenton. Conferência nacional sobre a qualidade do ambiente, 6, Lisboa, 1999 - "Actas da 6. ${ }^{a}$ Conferência Nacional sobre a Qualidade do Ambiente". Lisboa: Universidade Nova, 1999. v. 3, p. 475-479. 
SÁNCHEZ PERÉZ, J. A.; CARRA, I.; SIRTORI, C.; AGÜERA, A.; ESTEBAN, B. Fate of thiabendazole through the treatment of a simulated agro-food industrial effluent by combined MBR/Fenton processes at $\mu \mathrm{g} / \mathrm{L}$ scale. Water Res., v. 51, p. 55-63, 2014.

ZORPAS, A. A.; COSTA, C. N. Combination of Fenton oxidation and composting for the treatment of the olive solid residue and the olive mile wastewater from the olive oil industry in Cyprus. Bioresour. Technol., v. 101(20), p. 7984-7987, 2010. 